\title{
Understanding segregation and mixing effects in a riser using the quadrature method of moments
}

\author{
A. Dutta, J. Raeckelboom, G. J. Heynderickx \& G. B. Marin \\ Laboratorium voor Chemische Technologie, Ghent University, Belgium
}

\begin{abstract}
Segregation and mixing effects of particle diameter distributions are numerically investigated in the riser section of a circulating fluidized bed. A granular kinetic theory based approach, which implements the Eulerian quadrature-based moment method to describe the particle phase, is coupled to an Eulerian multi-fluid solver through a population balance model. The gas-solid multiphase flow is two-dimensional, transient and isothermal. The particle distributions fed from each side inlet of the riser have different variances, but the same mean diameter. The core-annular regime used as a numerical benchmark for riser flows is well predicted. A comparison in the homogeneity of particle mixing is made from the lower-order moments of the particle distribution obtained at various positions and at different axial lengths along the riser. It is seen that the relative standard deviation of the particle distribution varies spatially, indicating dynamic mixing inside the riser.
\end{abstract}

Keywords: mixing, riser, multiphase, quadrature method of moments.

\section{Introduction}

Multiphase flows involving solid particles in contact with a carrier gas in circulating fluidized beds (CFB) are ubiquitous in most chemical, petrochemical and pharmaceutical industries. Gas-fluidized circulating beds are commonly used in coal combustion, the catalytic cracking of crude oil, pharmaceutical granulation, etc. Quantitative understanding of the hydrodynamics of fluidization is needed for the design and scale-up of these processes. In these processes, the knowledge of particle segregation and mixing is limited. Nevertheless, there are applications in which it is important to avoid particle segregation, e.g. in 
catalytic fluidized bed reactors, whereas in other applications it is necessary to achieve complete segregation or mixing, e.g. in fluidized bed combustors [1]. Usually, segregation is referred to as the spatial (both axial and radial) distribution of particles of different size and/or density. Dependence on the size and/or density differences is linked to operating conditions [2]. In a CFB riser, the solids size and/or density distribution gives rise to radial segregation resulting in a core-annulus structure, in which particles flow downwards along the walls in clusters while strands of particles move upwards, together with the dispersed particles in the centre [3]. The single-particle terminal velocity is often a decisive parameter affecting axial segregation in fluidized beds [4], while coreannulus flow is attributed to the radial segregation of the particles. Karri and Knowlton [5] experimentally found that the particle diameter distribution in the core is smaller than in the annulus due to shear and recirculation/backmixing effects generated from the solids downflow near the wall. Numerical studies using computational fluid dynamics (CFD) on solids segregation/mixing have been reported for binary, ternary [6,7] and even quaternary [8] mixtures of particles of varying size/density. To simulate a realistic particle distribution, the approach followed by most researchers $[9,10]$ is the use of discrete approximations of the continuous size distribution function. However, this sectional approach is not feasible when the distribution is broad as it requires a large number of discrete sizes to accurately represent the size distribution leading to high computational costs even for relatively simple situations. Recently, Fan and Fox [11] have implemented a direct quadrature-based moment method to simulate segregation phenomena for a continuous distribution, thus avoiding the need for discrete approximations. In this method, the particle distribution is represented through a finite number of nodes, commonly referred to as abscissas, in the quadrature method. The evolution of these nodes is tracked through the lower-order moments of the distribution. As such, only a few nodes suffice to represent the entire distribution thus representing an attractive alternative to the traditional discrete approaches [11]. In the present study, the mixing effect of particle diameter distributions in a riser is numerically investigated using the quadrature method of moments (QMOM) in a multi-fluid CFD code, based on an Eulerian-Eulerian approach. The influence of polydispersity on the core-annulus flow profile is discussed. The standard deviation and mean determined through the lower-order moments of the distributions are calculated at various positions and at different axial lengths along the riser. These are used to study the influence of the particle distribution on the overall flow behavior and in particular, the mixing and segregation effects in the riser.

\section{Numerical approach}

A multi-fluid model based on the Eulerian-Eulerian approach is used. The conservation equations are solved for each phase in the Eulerian frame. The gas phase is considered as the primary phase, whereas the solid phase is considered as secondary or dispersed phase. For the gas phase the mass and momentum conservation equations are Reynolds averaged. The effects of turbulence are 
taken into account via a dispersed $k-\varepsilon$ turbulence model, which is an extension of the single-phase $k-\varepsilon$ model [12], adapted for gas-solid interactions. For the solid phase, the transport equations for mass, momentum and granular temperature are obtained via the kinetic theory of granular flow (KTGF) [13].

\subsection{Population balance model (PBM)}

The general form of a particle population balance conservation equation, as written by Ramkrishna [14] is:

$$
\frac{\partial}{\partial t}\left[\rho_{s} n(x ; t)\right]+\nabla \cdot\left[\rho_{s} n(x ; t) \vec{u}_{s}\right]=\rho_{s} S(x ; t)
$$

where

$$
S(x ; t)=B(x ; t)-D(x ; t) .
$$

In eq. 1 , the number of particles, $n$, is distributed with respect to some intrinsic parameter, $x$ (say particle size). The variables $\rho_{s}$ and $\vec{u}_{s}$ refer to the density and velocity vector of the particles respectively. The source terms $B$ and $D$ represent birth and death rates due to aggregation, breakage, etc. Several approaches for solving these equations are available, the most common being the discrete approach. However, a computationally attractive approach is the method of moments in which the population balance is formulated in terms of the lowerorder moments in closed form [15]. For a homogeneous system, considering the number density function $n(x ; t)$ in terms of the particle size length (i.e. $x \equiv L$ ), the $k^{\text {th }}$ moment is defined by:

$$
m_{k}(t)=\int_{0}^{\infty} L^{k} n(L ; t) d L \quad k=0,1, \ldots ., 2 \mathrm{~N}-1 .
$$

It is important to note that, usually, $L$ is referred to as an internal coordinate in contrast to $x$ and $t$, which are external coordinates. A number of lower-order moments are sufficient to represent the particle number density, total surface area and total volume of the particles. The resulting moment transport equation obtained by applying the above moment transform to eq. 1 is:

$$
\frac{\partial}{\partial t}\left(\rho_{s} m_{k}\right)+\nabla \cdot\left(\rho_{s} u_{s} m_{k}\right)=\rho_{s}\left(\bar{B}_{k}-\bar{D}_{k}\right) \text {. }
$$

A major drawback of this method is the need for an exact closure model for the source contributions. An exact closure is only available for constant or simple linear forms of the aggregation kernel and size-independent growth [15]. This constraint is, however, avoided by replacing the exact closure by an approximation of the unclosed terms using an ad hoc Gaussian quadrature formula.

\subsubsection{The quadrature approximation (or Gaussian quadrature)}

In order to solve the transport equations (eq. 3) for the moments, a Gaussian quadrature is used to approximate the integral (eq. 2) by a finite summation of the products of weight, $\omega$ and abscissa, $L$ [15] as:

$$
m_{k}(t)=\int_{0}^{\infty} L^{k} n(L ; t) d L \approx \sum_{i=1}^{N} \omega_{i} L_{i}^{k}
$$


Thus a quadrature approximation of order $N$ is defined by its $N$ weights $\omega_{j}$ and abscissas $L_{j}$ and can be calculated from the first $2 N$ moments $m_{0}, m_{1} \ldots, m_{2 N-1}$ by writing the recursive relationship for the polynomials in terms of the moments $m_{k}$. From a practical point of view, the system is ill-conditioned because the direct solution would require a non-linear search. McGraw [16] recommends the use of Product Difference (PD) algorithm, first described by Gordon [17]. The PD algorithm requires only the moments as inputs and gives the weights and abscissas as output. This algorithm is based on the minimization of the error introduced by replacing the integral in eq. (2) with its quadrature approximation by deriving a tridiagonal matrix of rank $N / 2$ and finding its eigenvalues and eigenvectors.

\subsection{Coupling CFD and PBM}

The population balance model is coupled to the hydrodynamic model through a Sauter mean diameter, $d_{32}$, of the particle distribution given as:

$$
d_{32}=\frac{\sum N_{i} d_{i}^{3}}{\sum N_{i} d_{i}^{2}}=\frac{m_{3}}{m_{2}} .
$$

Here, $d_{32}$ is calculated from the ratio of the moments, $m_{3}$ and $m_{2}$. This value is then replaced with the particle diameter in the drag model and updated with every time step. The use of the Sauter mean diameter causes only a single set of Navier-Stokes equations to be solved. As such, the particle distribution and with it, the moments, will be transported at the same velocity of the solids phase. This implies that particles of different sizes will have the same velocity.

\section{System description}

The riser set-up used in the present study is similar to the one used in the challenge problem reported by Knowlton et al [18] and explained in detail by Benyahia et al $[19,20]$. Knowlton et al [18] presented their experimental data obtained from a CFB column riser with FCC particles at the $8^{\text {th }}$ International Fluidization Conference. They measured solids concentration and flux at given locations of the riser and observed core-annulus flow behavior along the riser height. The geometry of the $0.2-\mathrm{m}$ riser with a total height of $14.2 \mathrm{~m}$ is similar to the experimental set-up used by Knowlton et al. [18]. The bottom inlet of the riser is a gas distributor yielding a superficial velocity of $5.2 \mathrm{~m} / \mathrm{s}$. Gas and solids are fed from two side inlets with a velocity of $0.476 \mathrm{~m} / \mathrm{s}$. The total solids volume fraction at the riser inlets is $40 \%$. Although the original geometry of the riser has a single solid inlet [18], the main reason for selecting a two-inlet geometry design is to obtain mixing effects in the riser entrance zone similar to the experimental results [19]. At the inlets, the velocity and volume fraction of both the phases are specified. At the outlet, only the pressure (atmospheric) is specified. At the walls, the gas velocities are set equal to zero by introducing a no-slip boundary condition. The partial-slip boundary condition for the solids at 
the wall is specified by applying an equation for the tangential velocity of the particles [21] using a specularity coefficient of 0.0001 . The value is justified because an increase in the specularity coefficient would result in a reduction of the solids volume fraction at the walls, causing the core-annulus behavior to disappear [22]. Two lognormal distribution functions with a mean average diameter, $d_{\text {ave }}$ of $77 \mu \mathrm{m}$ and a relative standard deviation, $\sigma / d_{\text {ave }}$ of 0.2 and 0.5 are implemented at the side-inlets. The corresponding $d_{32}$ of the inlet feed distribution are $83.3 \mu \mathrm{m}$ and $120.3 \mu \mathrm{m}$ respectively. This approach is also a check to ensure that the QMOM model returns the correct non-varying average diameter as the statistical mean. In the simulations, only the first six moments of the lognormal distributions are tracked (i.e. $\left.m_{0}, m_{1}, . ., m_{5}\right)$, which implies the use of a 3-node quadrature approximation (i.e. $N=3$ ).

\section{Results and discussion}

A two-dimensional transient model approach incorporating the kinetic theory for the solid particles is used in the commercial code Fluent, version 6.3, to simulate the gas-solids flow in the riser. A transient two-dimensional approach simulates the dynamic behavior of a multiphase flow in a Circulating Fluidized Bed (CFB) within a reasonable period of time [23]. The simulations are continued for $40 \mathrm{~s}$ of real time. The time-averaged distribution of the variables is then computed considering the last $30 \mathrm{~s}$ of the simulation. Both log-normal $\left(\sigma / d_{\text {ave }}=0.2,0.5\right)$ and monosize $(d=77 \mu \mathrm{m})$ particle distributions at the inlet are simulated for comparison with the experimental observations of Knowlton et al [18]. It is noteworthy to mention that the value of $77 \mu \mathrm{m}$ for the monosize distribution corresponds to the probabilistic mean diameter of the log-normal distribution to ensure a test-similarity. The flow patterns in the riser show a transient behavior of the solids volume fraction and the solids velocity profiles for both log-normal (fig. 1(i)(a) and 1(ii)(a)) and monosize (fig. 1(i)(b) and 1(ii)(b)) distributions. Note that fig. 1 corresponds to the lower and upper section of the riser respectively. In both the cases, the solids volume fraction is higher near the walls where the solids velocity is lower. Clusters are seen to be formed in the top of the riser and to flow downwards along the walls. In the centre of the riser, the solids velocity is considerably higher giving rise to core-annular flow: a dilute core region and a dense region near the walls. The core-annulus profile appears to be more uniform when the solids are log-normally distributed through their moments. This is probably due to the coupling of the gas-solid hydrodynamics with the population balance model through the Sauter mean diameter, $d_{32}$, which varies with the moments of the distribution. Figure 2 shows a comparison of the time-averaged solids density distribution $\left(\rho_{s} \varepsilon_{s}\right)$ at a height of $3.9 \mathrm{~m}$ from the bottom of the riser. The flow in the core is better predicted using a log-normal size distribution than a monosize distribution. The core width agrees reasonably well with the experimental data. However, the solids density at the wall is underpredicted, possibly due to inaccuracies in the applied wall boundary condition [20]. 


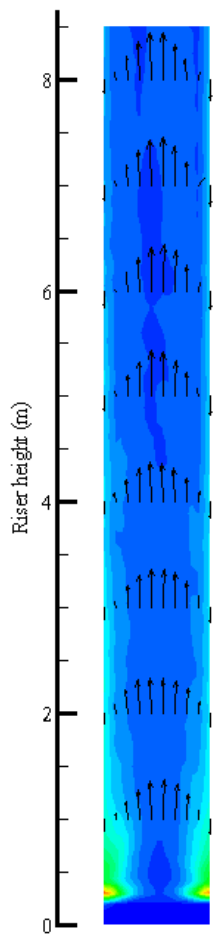

(a)

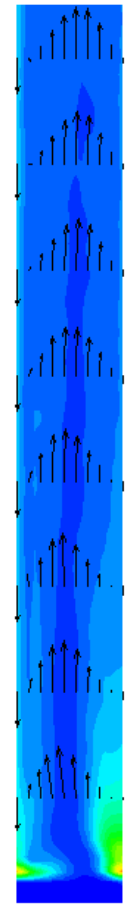

(b)

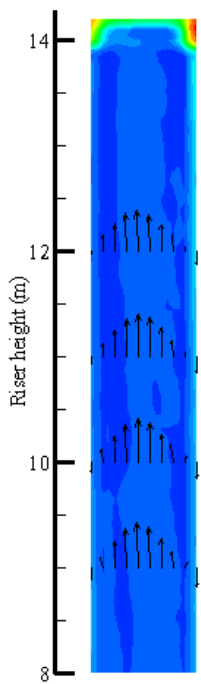

(a)

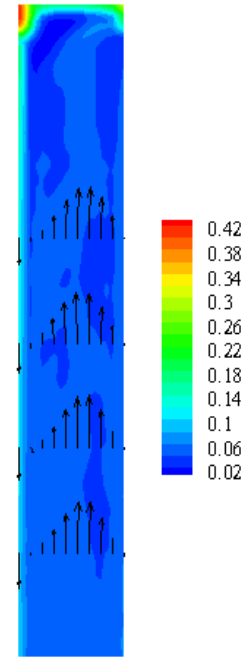

(b)

(i) Lower section

Figure 1: Comparison of the time-averaged (10-40s) solids volume fraction and velocity profiles for (a) log-normal and (b) monosize distribution, for the (i) lower $(0-8 \mathrm{~m})$ and (ii) upper $(8-14 \mathrm{~m})$ section of the riser.

Although the density of the solid particles used in the experimental set-up [18] is slightly different from the density of the particles used in the present study, a good qualitative fit for the time-averaged profile of the solids mass flux is obtained in the core of the riser when using a log-normal distribution. Similar to the experimental observations [18], the solids mass flux is found to be maximum at the central core of the riser (see fig. 3), although the solids density is at its lowest value there (see fig. 2). This is attributed with the core-annulus behavior i.e. with a high upward solids (and gas) axial velocity in the core and a low downward solids (and gas) axial velocity in the annulus of the riser. Thus a particle distribution captures a more realistic dynamic flow behavior in a gassolid riser. Figure 4 show a comparison of the time-averaged solids volume fraction profile for the log-normal and monosize distributions respectively. The effect of riser height on solids volume fraction is investigated by studying the profiles at $1 \mathrm{~m}, 3.9 \mathrm{~m}$ and $10 \mathrm{~m}$ from the bottom of the riser. Although a coreannular flow is observed in both the cases, there is a slight variation in the radial distribution of the solids volume fraction with the height of the riser. 


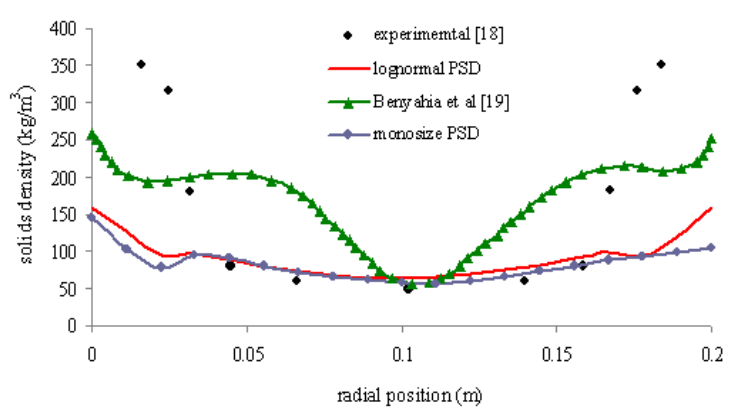

Figure 2: Time-averaged solids density distribution, using both log-normal and monosize distribution at $3.9 \mathrm{~m}$ from the bottom of the riser, compared with the numerical simulation [19] and experimental data [18].

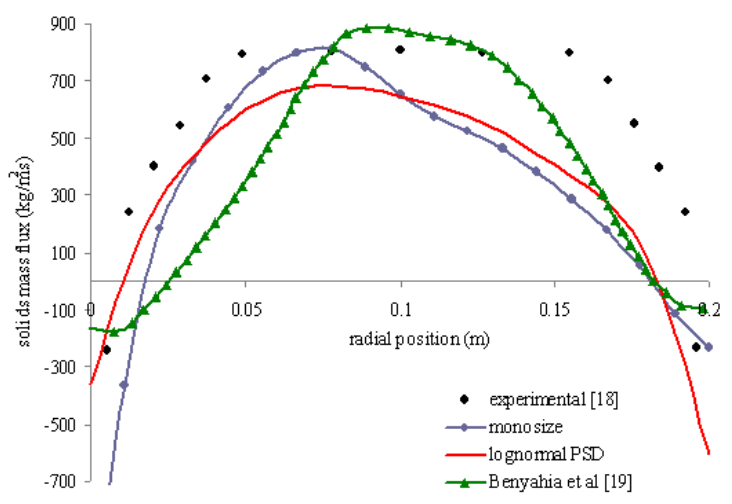

Figure 3: Time-averaged solids mass flux distribution using a log-normal distribution at $3.9 \mathrm{~m}$ from the bottom of the riser, compared with the numerical simulation [19] and experimental data [18].

The log-normal disribution almost maintains the core-annulus pattern higher up the riser (e.g. at $10 \mathrm{~m}$ ) whereas this pattern is assymetrically flattened in a monosize distribution. Indeed, the time-averaged profile of solids volume fraction differs remarkably along the height of the riser, as shown in fig. 4(b). A shift in the solids (and gas) axial velocity towards the right wall (see fig. 1(ii)) is indicated by a two-fold decrease in its solids volume fraction compared to the left wall. The difference in the solids flow behaviour in both the cases indicates that the particle distribution does, in fact, influence the gas-solid hydrodynamics in the riser.

A similar conclusion can be drawn from the time-averaged axial solids velocity profiles given in fig. 5 . 
188 Computational Methods in Multiphase Flow V

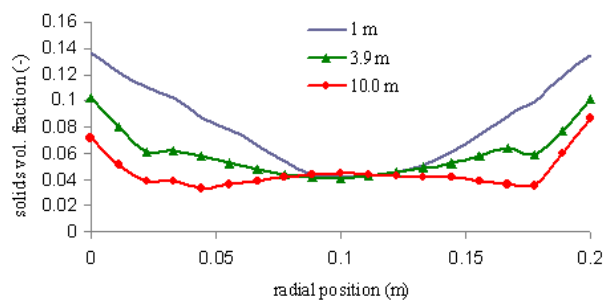

(a)

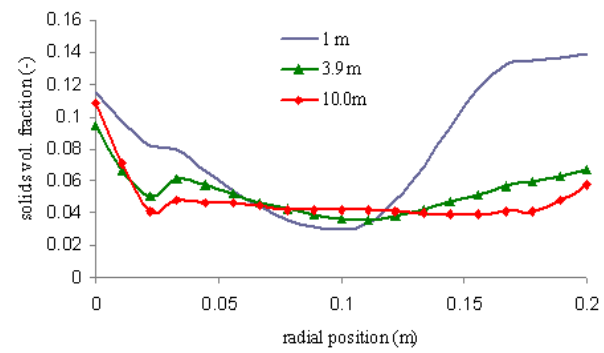

(b)

Figure 4: Effect of riser height on the time-averaged solids volume fraction profile in the riser for (a) log-normal and (b) monosize distribution.

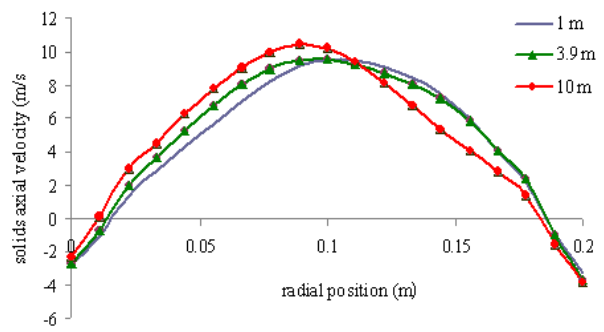

(a)

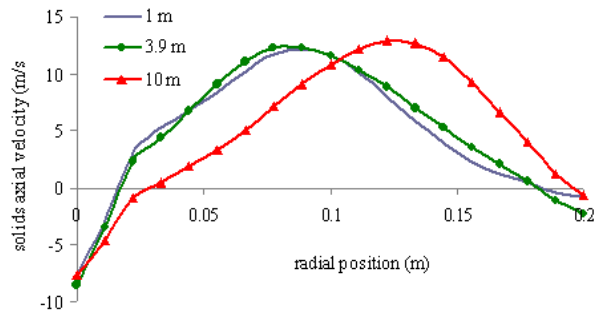

(b)

Figure 5: Effect of riser height on the time-averaged solids velocity profile in the riser for (a) log-normal and (b) monosize distribution. 
As seen in fig. 5, the peak of the solids axial velocity shifts slowly towards the wall along with the height of the riser, but in different directions. This shift in the peak of the the dilute core zone is slightly more for the monosize distribution (also see fig. 1). The influence of the particle distribution on the hydrodynamic behaviour in the riser can thus be clearly seen through the comparison in fig. 5 . At the wall, the solids have a negative axial velocity which indicates the downward movement of clusters along the walls. Remark that the calculated solids axial velocities are higher for the monosize distribution, due to a smaller width of the core.

To evaluate mixing of the particle distributions, a user-defined subroutine is written to obtain the values of the moments calculated for various positions and for multiple cross-sectional surfaces at different axial locations along the riser. The mean, $\mu$, and standard deviation, $\sigma$, is then calculated from the following:

$$
\begin{gathered}
\mu=\frac{m_{1}}{m_{0}} \\
\sigma=\sqrt{\frac{m_{2}}{m_{0}}-\left(\frac{m_{1}}{m_{0}}\right)^{2}} .
\end{gathered}
$$

Applying these formulas give the values of the mean and the standard deviation of the local and global distributions respectively. The standard deviation gives insight on how the particles are distributed over a surface depending on their size. The smaller the standard deviation (i.e. smaller range of different sizes), the more segregated is the particle distribution in the surface. The larger this value (i.e. larger range of different sizes), the more mixed is the distribution. Based on a standard deviation, it is possible to conclude whether two or more surface distributions are equally mixed or not. Through the calculation of the mean of the distribution for a surface, it is possible to determine differences in the sizes of the particles present in these surfaces. The mean, together with the standard deviation, are thus a measure of the radial segregation along the riser height. The results obtained at $1 \mathrm{~m}, 3.9 \mathrm{~m}$ and $10 \mathrm{~m}$ from the bottom of the riser are listed in table 1 and table 2 respectively at a simulated time of $40 \mathrm{~s}$. Note that the left and right positions in table 1 refer to 5 $\mathrm{mm}$ radial distance from the left and the right wall of the riser respectively, while the middle refers to the mid-point of the cross-sectional surface. The mixing and segregation of the lognormal distributions and the effect on the riser hydrodynamics can be observed from the results of table 1 and 2 . From table 1 , it can be concluded that the radial mixing of particles over a cross section of the riser along the height of the riser is very dynamic. At a height of $1 \mathrm{~m}$, the two distributions are mixing in the middle because the standard deviation of the distribution in the middle lies between the values of those at the walls. Higher up the riser i.e. at $3.9 \mathrm{~m}$ and further at $10 \mathrm{~m}$ from the bottom, it is observed that the distribution in the middle becomes more segregated, meaning that only a fraction of the particles introduced in the riser resides in the middle part of the riser. This also indicates the formation of a core-annular regime higher up the riser. 
Table 1: $\quad$ Estimation of the local particle diameter distribution over a surface for three positions along the riser height.

\begin{tabular}{|c|c|c|c|}
\hline \multirow{3}{*}{$\begin{array}{l}\text { Height from the bottom of } \\
\text { the riser }(\mathrm{m})\end{array}$} & \multicolumn{3}{|c|}{ Radial position } \\
\hline & Left & Middle & Right \\
\hline & \multicolumn{3}{|c|}{ Average diameter, $\mu_{\text {local }}$} \\
\hline 1.0 & $7.70 \times 10^{-5}$ & $7.70 \times 10^{-5}$ & $7.70 \times 10^{-5}$ \\
\hline 3.9 & $7.70 \times 10^{-5}$ & $7.70 \times 10^{-5}$ & $7.70 \times 10^{-5}$ \\
\hline \multirow[t]{2}{*}{10.0} & $7.70 \times 10^{-5}$ & $7.70 \times 10^{-5}$ & $7.70 \times 10^{-5}$ \\
\hline & \multicolumn{3}{|c|}{ Standard deviation, $\sigma_{\text {local }}$} \\
\hline 1.0 & $3.72 \times 10^{-5}$ & $3.41 \times 10^{-5}$ & $3.01 \times 10^{-5}$ \\
\hline 3.9 & $3.52 \times 10^{-5}$ & $2.75 \times 10^{-5}$ & $3.02 \times 10^{-5}$ \\
\hline \multirow[t]{2}{*}{10.0} & $3.25 \times 10^{-5}$ & $2.96 \times 10^{-5}$ & $3.01 \times 10^{-5}$ \\
\hline & \multicolumn{3}{|c|}{ Spread ratio, $\sigma_{\text {local }} / \sigma_{\text {local }}^{\prime}$} \\
\hline 1.0 & 1.0 & 0.92 & 0.81 \\
\hline 3.9 & 1.0 & 0.78 & 0.86 \\
\hline 10.0 & 1.0 & 0.91 & 0.93 \\
\hline
\end{tabular}

Table 2: $\quad$ Estimation of the global particle diameter distribution for different radial surfaces along the riser height.

\begin{tabular}{|l|l|l|l|}
\hline \multirow{2}{*}{ Parameters of distribution } & \multicolumn{3}{|l|}{ Height from the bottom of the riser $(\mathrm{m})$} \\
\cline { 2 - 4 } & 1.0 & 3.9 & 10.0 \\
\hline Average diameter, $\mu_{\text {global }}$ & $7.70 \times 10^{-5}$ & $7.70 \times 10^{-5}$ & $7.70 \times 10^{-5}$ \\
\hline Standard deviation, $\sigma_{\text {global }}$ & $3.37 \times 10^{-5}$ & $2.93 \times 10^{-5}$ & $3.02 \times 10^{-5}$ \\
\hline Spread ratio, $\sigma_{\text {global }} / \sigma_{\text {global }}^{\prime}$ & 1.0 & 0.87 & 0.89 \\
\hline Rel. std. deviation, $\sigma_{\text {global }} / \mu_{\text {global }}$ & 0.438 & 0.38 & 0.392 \\
\hline
\end{tabular}

The surface-averaged mean and standard deviation of the lognormal distributions are given in table 2 for surfaces at different radial cross-sections along the height of the riser.

It can be concluded that the distribution of particles along the riser height show segregation which depends on the hydrodynamic conditions of the riser. The correspondence of the local and the global mean with the mean average diameter of the distributions indicates the robustness of the QMOM model in returning the correct average diameter for multiple distributions with a varying standard deviation. These observations confirm that the gas-solid simulation in a riser using particle distributions show segregation and mixing effects, which is influenced by the overall riser hydrodynamics.

\section{Conclusion}

The Quadrature-based Method of Moment (QMOM) has been successfully applied to solve the evolution of a continuous particle distribution in a multi- 
fluid CFD model for flows in a gas-solid riser. The core-annulus profile usually observed in riser flow is well predicted by this model. The simulation results considering a log-normal particle diameter distribution give a better qualitative fit with the experimental data as compared to the fit when using a monosize distribution. In comparison with the results for a monosize distribution, the coreannulus profile is much more uniform and maintained higher up in the riser for the log-normal distribution. It can be said that polydispersity has an influence on the gas-solid hydrodynamics of a riser, especially on the core-annulus flow profile. The radial segregation and mixing for the particle distributions, calculated for various positions and for multiple cross-sectional surfaces at different axial locations along the riser, is evaluated from the local and surfaceaveraged moments of the distribution. It is seen that the radial mixing profile is dynamic as it changes along the height of the riser. Although the distributions are quite mixed in the middle of a cross-sectional surface close to the inlet, it becomes more segregated as it approaches the outlet. The effect of mixing homogeneity due to particles size distribution on the hydrodynamic conditions of a riser is investigated. Spatial segregation of particles can be observed if, according to their size, different velocities of the particles are computed. For this, a modification in the quadrature-based moment method is necessary.

\section{References}

[1] Das, M., Banerjee, M. \& Saha, R.K., Segregation and mixing effects in the riser of a circulating fluidized bed. Powder Technology, 178(3), pp. 179186, 2007.

[2] Nakagawa, N., Dingrong Bai, Shibuya, E., Kinoshita H., Takarada, T. \& Kato, K., Segregation of particles in binary solids circulating fluidized beds. Journal of Chemical Engineering of Japan, 27(2), pp. 194-198, 1994.

[3] Bader, R., Findlay, J. \& Knowlton, T., Gas/solid flow patterns in a 30.5-cm diameter circulating fluidized Bed. Circulating Fluidized Bed Technology: II, Basu, P. and Large, J. F. (eds.), Pergamon Press, p. 123, 1988.

[4] Hirschberg, B. \& Werther, J., Factors affecting solids segregation in circulating fluidized-bed riser. AIChE Journal, 44(1), pp. 25-34, 1998.

[5] Karri, S.B.R. \& Knowlton, T.M., Flow direction and size segregation of annulus solids in a riser In: L.S. Fan and T.M. Knowlton, Editors, Fluidization IX, Engineering Foundation, New York, pp. 189-194, 1998.

[6] Iddir, H., Arastoopour, H. \& Hrenya, C.M., Analysis of binary and ternary granular mixtures behavior using the kinetic theory approach. Powder Technology, 151(1-3), pp.117-125, 2005.

[7] Huilin, L. \& Gidaspow, D., Hydrodynamics of binary fluidization in a riser: CFD simulation using two granular temperatures. Chemical Engineering Science, 58(16), pp. 3777-3792, 2003.

[8] Nienow, A.W., Naimer, N.S. \& Chiba, T.S., Studies of segregation/mixing in fluidized beds of different size particles. Chemical Engineering Communications 62(1-6), pp. 53-66, 1987. 
[9] Mathiesen, V., Solberg, T. \& Hjertager, B.H., Predictions of gas/particle flow with an Eulerian model including a realistic particle diameter distribution. Powder Technology 112(1-2) pp. 34-45, 2000.

[10] van Wachem, B.G.M., Schouten, J.C., van den Bleek, C.M., Krishna, R. \& Sinclair, J.L., CFD modeling of gas-fluidized beds with a bimodal particle mixture. AIChE Journal, 47(6), pp.1292-1302, 2001.

[11] Fan, R \& Fox, R., Segregation in polydisperse fluidized beds: Validation of a multi-fluid model. Chemical Engineering Science 63(1) pp.272 -285, 2008.

[12] Hinze, J., Turbulence: An Introduction to its Mechanism and Theory. McGraw-Hill, New York, 1959.

[13] Gidaspow, D., Multiphase Flow and Fluidization, Continuum and Kinetic Theory Descriptions. Academic Press, New York, 1994.

[14] Ramkrishna, D., Population balances. Theory and applications to particulate systems in engineering. Academic Press, New York, 2000.

[15] Marchisio, D.L., Pikturna, J.T., Fox, R.O., Vigil, R.D. \& Barresi, A.A., Quadrature method of moments for population-balance equations. AIChE Journal, 49(5), pp. 1266-1276, 2003.

[16] McGraw, R., Description of Aerosol Dynamics by the Quadrature Method of Moments, Aerosol Sci. Tech. 27(2), pp. 255-265,1997.

[17] Gordon, R. G., Error Bounds in Equilibrium Statistical Mechanics. J. Math. Phys., 9, pp.655-663, 1968.

[18] Knowlton, T., Geldart, D., Matsen, J. \& King, D. Comparison of CFB Hydrodynamic Models. PSRI Challenge Problem, $8^{\text {th }}$ International Fluidization Conference, Tour, France, May, 1995.

[19] Benyahia, S., Arastoopour, H. \& Knowlton, T. M. Two-dimensional transient numerical simulation of solids and gas flow in the riser section of a circulating fluidized bed. Chemical Engineering Communications, 189(4), pp. 510-527, 2002.

[20] Benyahia, S., Arastoopour, H., Knowlton, T.M. \& Massah, H., Simulation of particles and gas flow behavior in the riser section of a circulating fluidized bed using the kinetic theory approach for the particulate phase. Powder Technology, 112(1-2), pp. 24-33, 2000.

[21] Johnson, P.C. \& Jackson, R., Frictional-collisional constitutive relations for granular materials, with application to plane shearing. Journal of Fluid Mechanics 176, pp. 67-93, 1987.

[22] Benyahia S., Syamlal M. \& O'Brien, T.J. Study of the ability of multiphase continuum models to predict core-annulus flow. AIChE Journal, 53(10), pp.2549-2568, 2007.

[23] Tsuo, Y. P. \& Gidaspow, D., Computation of flow patterns in circulating fluidized beds. AIChE Journal, 36(6), pp. 885-896, 1990. 